\title{
Mapping Asian anthropogenic emissions of non-methane volatile organic compounds to multiple chemical mechanisms
}

M. Li et al.

Correspondence to: Q. Zhang (qiangzhang@tsinghua.edu.cn) 
Table S1. 2006 Asian total NMVOC emissions and source profiles*

\begin{tabular}{|c|c|c|c|c|}
\hline Sector & Fuel/Product & Profile used in this work & $\begin{array}{l}\text { Profiles used in the } \\
\text { original INTEX-B }\end{array}$ & $\begin{array}{l}\text { NMVOC } \\
\text { Emissions /Gg }\end{array}$ \\
\hline \multirow[t]{2}{*}{ Power } & Coal & 1178 & 1178 & 1130.7 \\
\hline & Biofuel & $\begin{array}{l}\text { Tsai et al. (2003), Liu et al. } \\
\text { (2008), Wang et al. (2009), } \\
\text { 1167, 4420, 4421, } 5561 \\
\text { (Andreae and Merlet, } \\
\text { 2001) }\end{array}$ & Tsai et al. (2003) & 27.2 \\
\hline Industry & Coal/Boiler, Kilns & Liu et al. (2008), 1185 & 1185 & 1701.2 \\
\hline \multirow[t]{3}{*}{ Combustion } & Coke Oven Blast Furnace & 5 & 217 & 297.7 \\
\hline & Gas/Boiler & & & \\
\hline & Biofuel/Boiler & $\begin{array}{l}\text { Tsai et al. (2003), Liu et al. } \\
\text { (2008), Wang et al. (2009), } \\
\text { 1167, 4420, 4421, } 5561 \\
\text { (Andreae and Merlet, } \\
\text { 2001) }\end{array}$ & Tsai et al.(2003) & 16.8 \\
\hline Industry & Coke & 11,217 & 11 & 477.6 \\
\hline \multirow[t]{24}{*}{ Non-combustion } & Iron/Steel & 13,16 & 14 & 198.5 \\
\hline & Asphalt Roofing & 23,24 & 24 & 45.0 \\
\hline & Pharmaceutical Solvent & 3040, 3144 & 79 & 291.5 \\
\hline & Asphalt Production & Liu et al. (2008) & 1007 & 61.7 \\
\hline & Paint Production & 1094 & 1094 & 113.7 \\
\hline & Printing Ink & 72 & 72 & 34.7 \\
\hline & Glue Production & 2423 & 2423 & 133.7 \\
\hline & Tire & $272,273,274$ & 274 & 277.8 \\
\hline & Rubber & 1008 & 1008 & 84.3 \\
\hline & PVC & 1091 & 1091 & 434.2 \\
\hline & Polystyrene & 1004 & 1004 & 393.6 \\
\hline & Pulp/Paper Production & 4730, 4731, 4732 & 1189 & 107.1 \\
\hline & Ammonia & 2420 & 2420 & 241.2 \\
\hline & Black Carbon & 1002 & 1002 & 199.0 \\
\hline & Ethylene & 2461 & 2461 & 88.4 \\
\hline & Polyethylene & 2547 & 2547 & 91.6 \\
\hline & Styrene & $1066,1067,1068$ & 1066 & 1.5 \\
\hline & Polystyrene & 1004 & 1004 & 6.6 \\
\hline & VinylCl & 1091 & 1065 & 32.5 \\
\hline & PVC & 1091 & 1091 & 35.9 \\
\hline & Polypropylene & 68 & 68 & 129.7 \\
\hline & Storage & 1055, 1069, 1087 & 2470 & 319.5 \\
\hline & NG Production and Handle & 2442 & 2442 & 128.1 \\
\hline & $\begin{array}{l}\text { Crude Oil Production and } \\
\text { Refinery }\end{array}$ & $\begin{array}{l}\text { Liu et al. (2008), 296, 305, } \\
\text { 1207, 1208, 1212, } 2487\end{array}$ & 297 & 1481.7 \\
\hline
\end{tabular}




\begin{tabular}{|c|c|c|c|c|}
\hline & Gasoline Deposition & Liu et al. (2008) & 1190 & 1691.7 \\
\hline & Diesel Deposition & Liu et al. (2008) & 1011 & 3.7 \\
\hline & Food and Drink & 8538 & 8538 & 924.6 \\
\hline & Fabrics & 1092, 1095, 1096 & 1096 & 285.0 \\
\hline & Waste Treatment & $122,202,3002$ & 202 & 85.3 \\
\hline & New Vehicle Varnish Paint & Yuan et al. (2010) & 2546 & 211.8 \\
\hline & Vehicle Refurnish Paint & Yuan et al. (2010) & 2402 & 226.7 \\
\hline & Other Industry Varnish & 1003, 1013, 1016 & 2420 & 2444.3 \\
\hline & Paint & & & \\
\hline & Printing Ink & $\begin{array}{l}\text { Yuan et al. (2010), 182, } \\
\text { 183, 1191, 2543, } 2544\end{array}$ & 2545 & 156.6 \\
\hline & Passenger Vehicle & 1194 & 2493 & 50.0 \\
\hline & Treatment Solvent & & & \\
\hline & Wood Treatment Solvent & 2404 & 3087 & 73.8 \\
\hline & $\begin{array}{l}\text { Printing Cleaning Gasoline } \\
\text { Solvent }\end{array}$ & Liu et al. (2008), 1195 & 1022 & 430.3 \\
\hline $\begin{array}{l}\text { Residential } \\
\text { Combustion }\end{array}$ & Coal/Boiler, Stoves & $\begin{array}{l}\text { Tsai et al. (2003), Liu et al. } \\
\text { (2008) }\end{array}$ & 1185 & 566.5 \\
\hline & Biofuel & $\begin{array}{l}\text { Tsai et al. (2003), Liu et al. } \\
\text { (2008), Wang et al. (2009), } \\
\text { 1167, 4420, 4421, } 5561 \\
\text { (Andreae and Merlet, } \\
\text { 2001) }\end{array}$ & Tsai et al.(2003) & 15649.7 \\
\hline $\begin{array}{l}\text { Residential } \\
\text { Non-combustion }\end{array}$ & Paint Use & $\begin{array}{l}\text { Na et al. (2004), Liu et al. } \\
\text { (2008), Yuan et al. (2010) }\end{array}$ & 1013 & 2692.8 \\
\hline & Domestic Solvent Use & 197,8500 & 8500 & 2349.1 \\
\hline & Dry Clean C2Cl4 Solvent & 1193 & 1193 & 163.7 \\
\hline On-road & $\mathrm{MC}$ & Liu et al. (2008) & 1203 & 8447.8 \\
\hline Transportation & $\begin{array}{l}\text { Light Duty Gasoline } \\
\text { Vehicle }\end{array}$ & $\begin{array}{l}\text { Duffy et al. (1999), Liu et } \\
\text { al. (2008), 1203, } 4556 \\
\text { (Schauer et al., 2002) }\end{array}$ & 1203 & 4370.2 \\
\hline & $\begin{array}{l}\text { Heavy Duty Gasoline } \\
\text { Vehicle }\end{array}$ & $\begin{array}{l}\text { Duffy et al. (1999), Liu et } \\
\text { al. (2008), 1186, 1203, } \\
4556 \text { (Schauer et al., 2002) }\end{array}$ & 1186 & 2559.6 \\
\hline & Diesel Vehicle & $\begin{array}{l}\text { Liu et al. (2008), } 4674 \\
\text { (Schauer et al., 1999) }\end{array}$ & 1201 & 784.6 \\
\hline Off-road & Diesel & Liu et al. (2008), 3161 & 3161 & 1762.0 \\
\hline Transportation & Air & 1099 & 1098 & 70.8 \\
\hline Others & Oil & 1,2 & 1 & 195.7 \\
\hline & Natural Gas & 3 & 3 & 33.5 \\
\hline
\end{tabular}

*Profiles taken from the SPECIATE 4.2 database are presented as corresponding "P_NUMBER"s in the database (Hsu and Divita, 2009; Simon et al., 2010). 


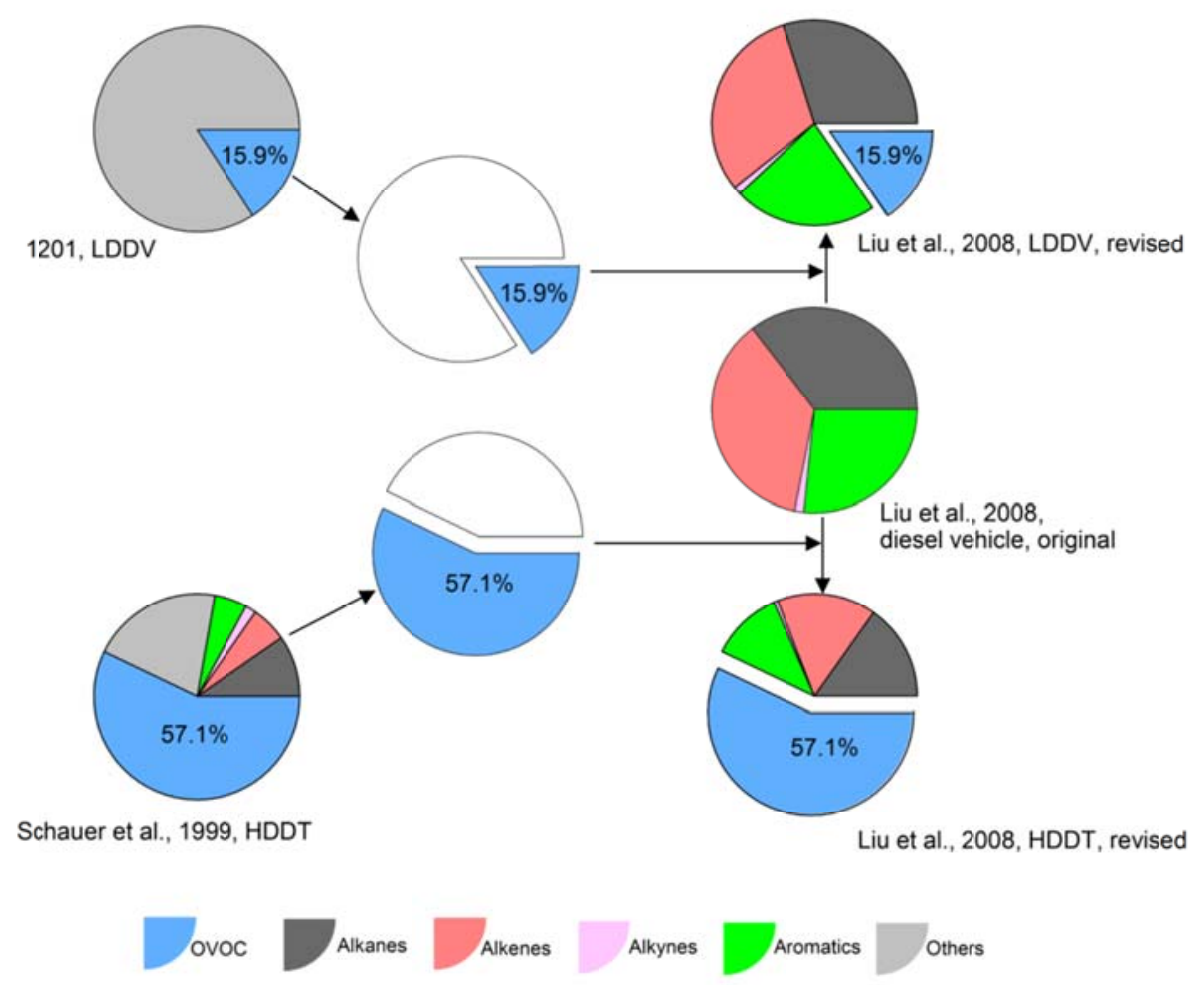

Figure S1. Inclusion of OVOC fraction for diesel truck profiles. LDDV: light duty diesel vehicles; HDDT: heavy duty diesel trucks. 1201 and 4674 are the "P_NUMBER"s of profiles in the SPECIATE 4.2 database 


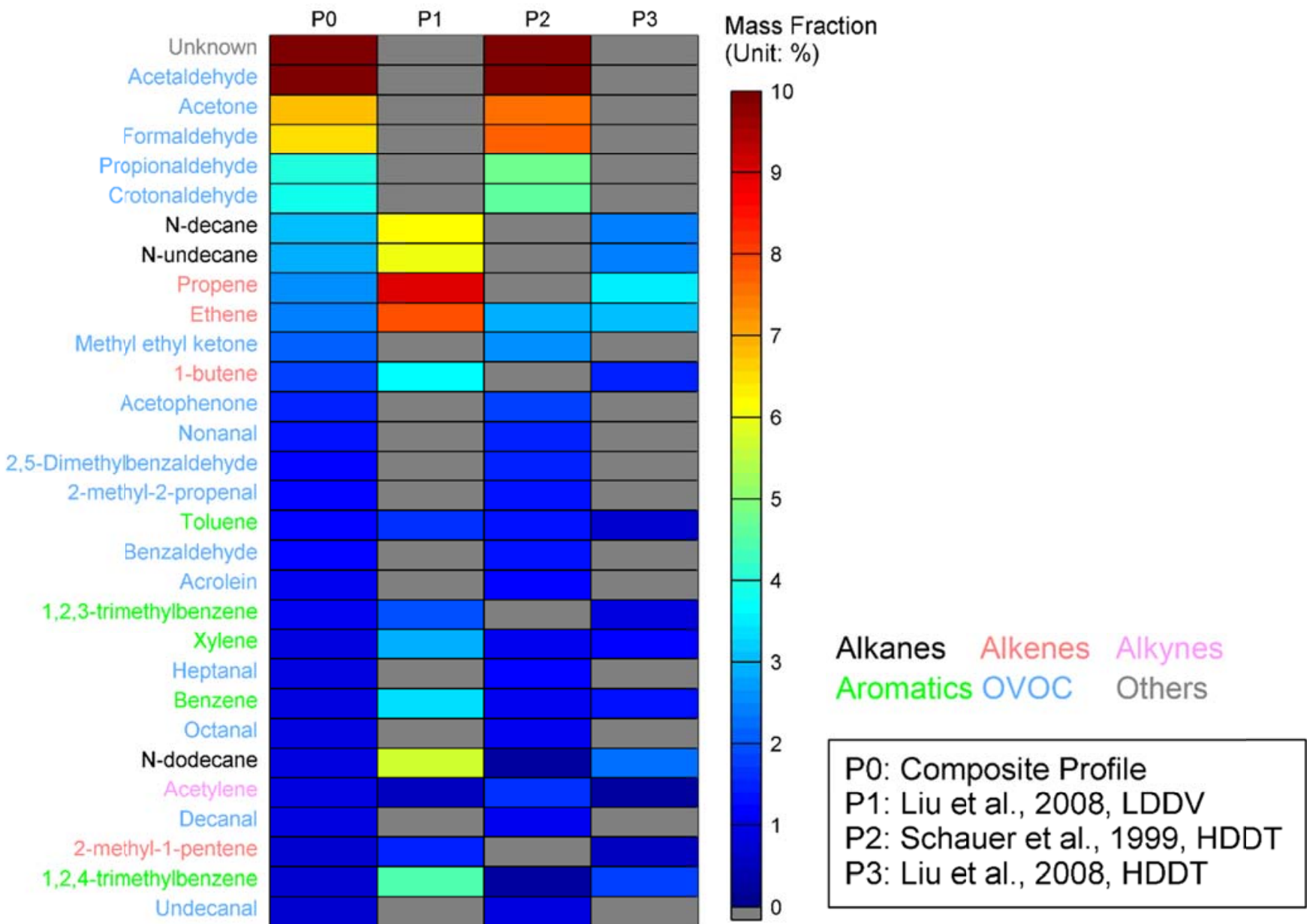

Figure S2. Sensitivity of emissions for individual species for the diesel vehicle source 


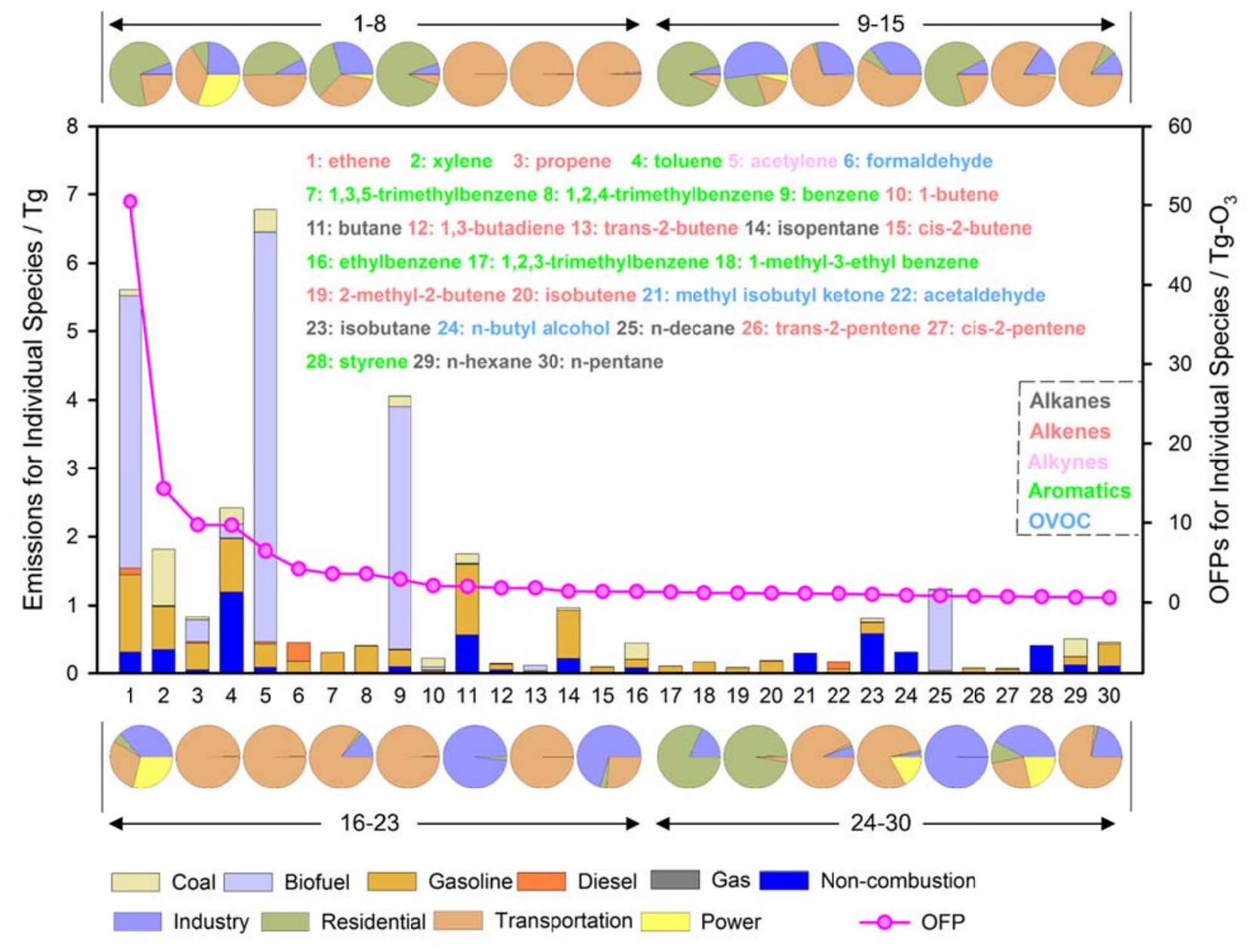

Figure S3. Emissions by individual NMVOC species and corresponding OFPs in the original INTEX-B emission inventory 


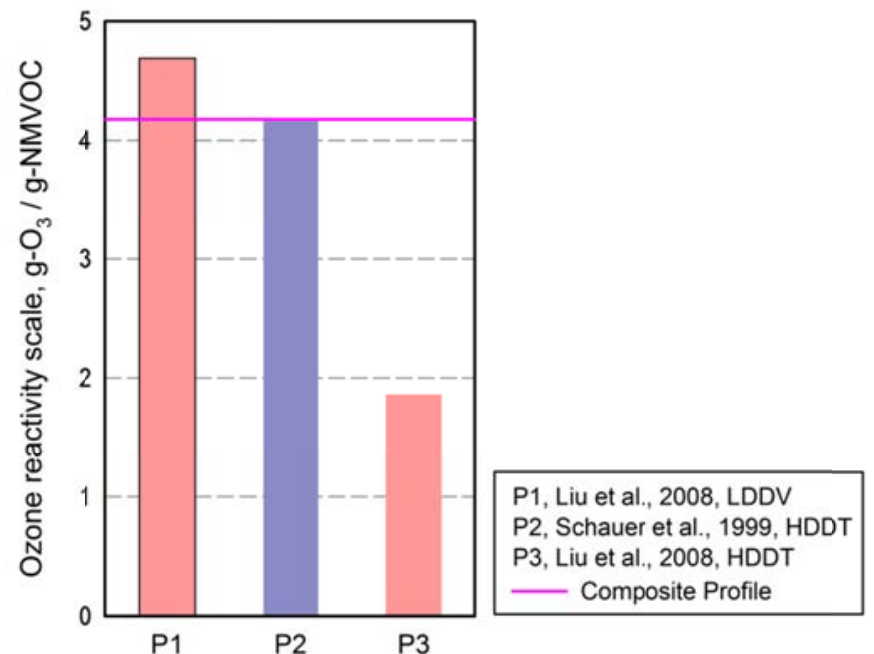

Figure S4. Sensitivity of OFPs to profile selection for the diesel vehicle source 

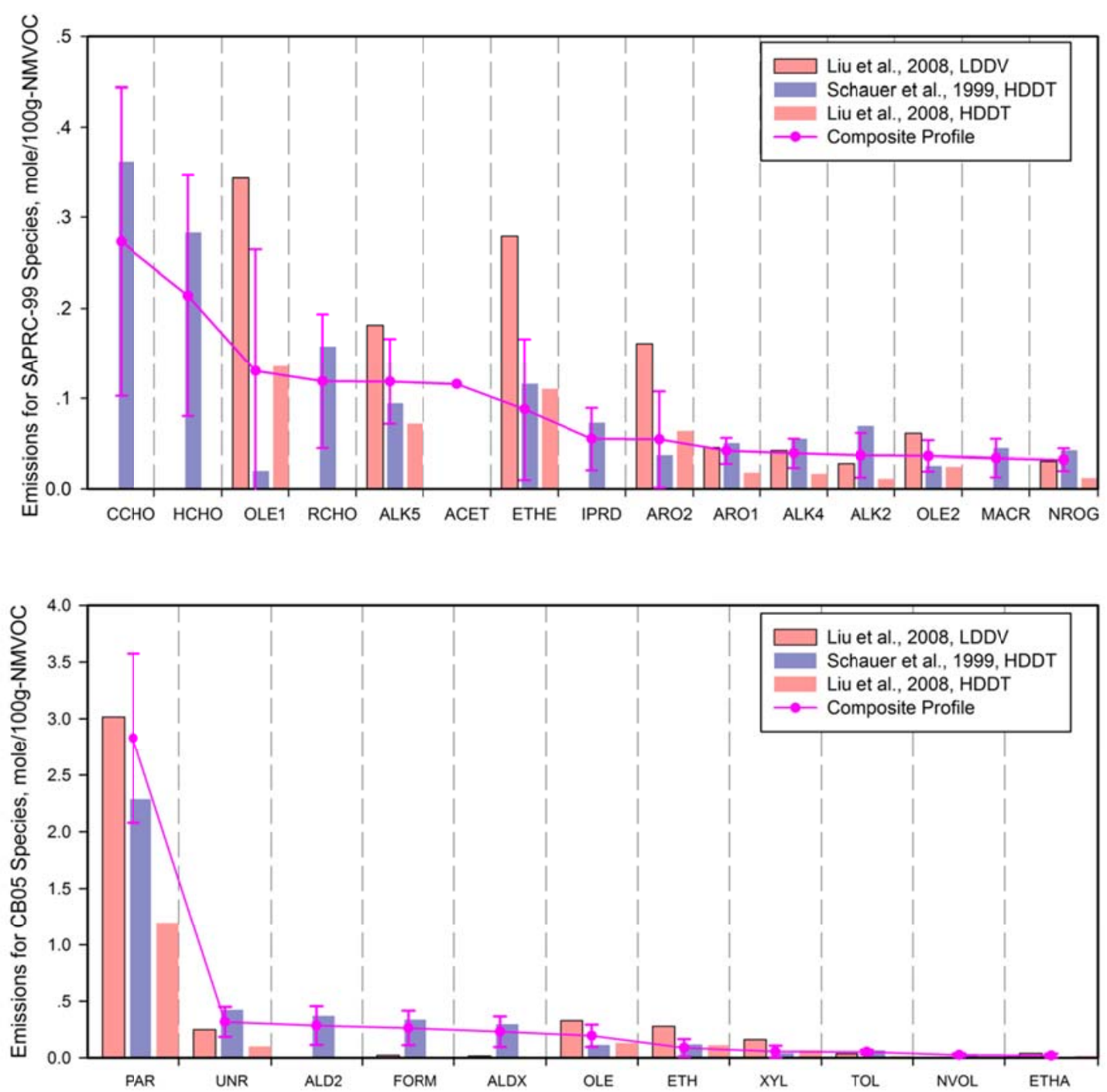

Figure S5. Sensitivity of model-ready emissions to profile selection for the diesel vehicle source 


\section{References}

Hsu, Y. and Divita, F.: SPECIATE 4.2, speciation database development documentation, final report, EPA/600-R-09/-38, 2009.

Simon, H., Beck, L., Bhave, P. V., Divita, F., Hsu, Y., Luecken, D., Mobley, J. D., Pouliot, G. A., Reff, A., Sarwar, G., and Strum, M.: The development and uses of EPA's SPECIATE database, Atmos. Pollut. Res., 1, 196-206, 2010. 